\title{
Análise de interfaces de máquinas de lavar roupas sob a ótica da linguagem gráfica
}

\section{Analysis of washing machine interfaces from the perspective of graphic language}

\author{
Juliana F. Oliveira, Dominique L. Adam, Juliana Bueno, Maria Lucia L. R. Okimoto
}

análise gráfica, design de interface, eletrodomésticos, acessibilidade

\begin{abstract}
As interfaces de eletrodomésticos apresentam uma configuração gráfico-informacional híbrida, que relaciona componentes verbais, pictóricos e esquemáticos para tornar perceptíveis visualmente as funções e ações possíveis de serem realizadas. Por meio de teclas e botões é possível acionar e selecionar comandos, já através de legendas e visores digitais é possível identificar diferentes categorias informacionais que nortearão as tomadas de decisão durante a interação. Considerando a acessibilidade gráfico-informacional dessas interfaces, o presente artigo tem como objetivo analisar, sob a perspectiva da linguagem gráfica, interfaces de máquinas de lavar roupas com o intuito de identificar padrões de estrutura e relação entre os componentes gráficos dessas interfaces. Os resultados preliminares deste estudo sugerem padrões da organização visual de interfaces de máquinas de lavar roupas que podem vir a orientar, em pesquisas futuras, a formatação de um modelo conceitual de interface que apresente alternativas sensoriais aos componentes visuais gráficos para possibilitar uma interação acessível para pessoas com deficiência visual.
\end{abstract}

graphic analysis, interface design, home appliances, accessibility

Home appliances interfaces present a hybrid graphic-informational configuration, which uses verbal, pictorial, and schematic components to visually identify possible functions and actions to be performed. Through keys, buttons it is possible to activate and select functions, and through subtitles and digital displays, it is possible to identify different information categories that will guide decision-making during the interaction. Considering the graphic-informational accessibility of these interfaces, this paper aims to analyze washing machine interfaces from the perspective of graphic language, in order to identify structural patterns and the relationship between the graphic components of these interfaces. The results of this study suggest patterns of the visual organization of these interfaces that may guide, in future research, the design of a conceptual model that presents sensory alternatives for the visual graphic components, to enable accessible interaction for the visually impaired.

\section{Introdução}

Nacionalmente, há uma ampla discussão acerca da acessibilidade de eletrodomésticos (Senado, 2019). Este fato vem de encontro com o que defende a Lei Brasileira de Inclusão da Pessoa com Deficiência Brasil (2015), em que todos devem possuir a mesma oportunidade de acesso à informação. Diante deste cenário, inicia-se um questionamento acerca do uso da linguagem gráfica em interfaces e de que forma esta pode ser apresentada para tornar as informações da interface do produto acessíveis às pessoas com deficiência visual.

Anais do $10^{\circ} \mathrm{CIDI}$ e $10^{\circ} \mathrm{CONGIC}$

Kelli C.A.S. Smythe, Rafael de Castro Andrade (orgs.)

Sociedade Brasileira de Design da Informação - SBDI

Curitiba | Brasil | 2021
Proceedings of the $10^{\text {th }} \mathrm{CIDI}$ and $10^{\text {th }} \mathrm{CONGIC}$

Kelli C.A.S. Smythe, Rafael de Castro Andrade (orgs.)

Sociedade Brasileira de Design da Informação - SBDI Curitiba | Brazil | 2021 
A linguagem gráfica é definida por Twyman (1979) por seu caráter intencionalmente visível em prol de comunicar uma mensagem, a partir dos modos verbal, pictórico e esquemático. No contexto do Design de Interação, a linguagem gráfica é o meio que permite que palavras, imagens, ícones, suas características e relações sejam estruturadas em informações perceptíveis aos usuários, depois, apresentadas através de um suporte físico ou digital em um contexto de uso específico (Campos et al, 2008; Shneiderman \& Plaisant, 2005).

A partir de pesquisas na área de acessibilidade para pessoas com deficiência e a evolução dos recursos assistivos, considera-se a abordagem do Design Inclusivo uma maneira de tornar possível soluções interativas que atendam a um maior número de usuários (Adam, 2020;

Aguiar, 2004; Alvarenga, 2016; Rezende, 2014; Raposo, 2015; Oliveira, 2018). Esta abordagem metodológica visa elevar a experiência de uso dos produtos e serviços através de uma conduta projetual centrada no usuário (Clarkson et al., 2007).

Atendendo o projeto de interfaces centrado no usuário, reitera-se a necessidade de analisar as capacidades humanas de processamento da informação e o contexto de uso como fatores orientadores para as escolhas projetuais, avaliando-os conforme a eficácia, eficiência e satisfação de uso - dimensões de usabilidade (Shneiderman \& Plaisant, 2005) (Figura 1).

Figura 1: Relação usuário-produto. Fonte: as autoras (2021) com base em Campos et al (2008) e Shneiderman e Plaisant (2005).

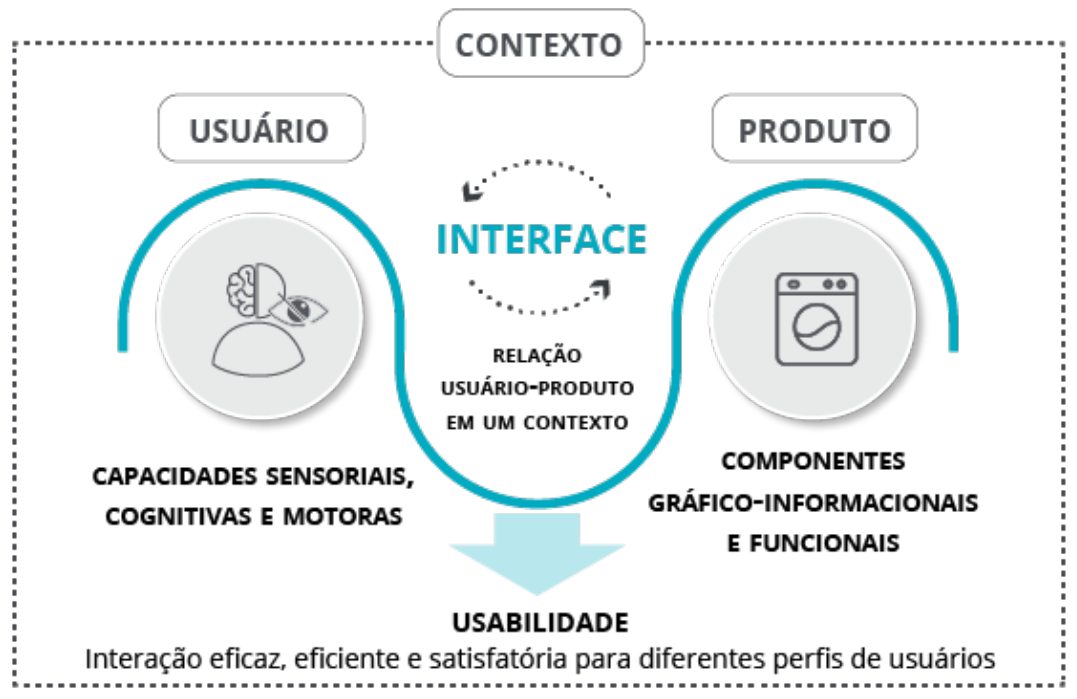

Considerando a automação de tarefas e das atividades domésticas a partir do uso de eletrodomésticos cada vez mais inteligentes e interativos, diversas pesquisas questionam a acessibilidade e a usabilidade desses produtos em relação aos usuários com deficiência visual. Informações majoritariamente disponibilizadas pelo uso da linguagem gráfica verbal, pictórica e esquemática (perceptíveis por meio do canal visual), sem correspondente em outra modalidade (e.g. sonoro ou tátil-vibracional) comprometem o uso do produto (Aguiar, 2004; Rezende, 2014; Raposo, 2015; Oliveira, 2018). 
À vista do contexto apresentado, esta pesquisa tem o objetivo de complementar os estudos de acessibilidade de interfaces de eletrodomésticos identificando os componentes gráficos, bem como suas relações, em interfaces de máquina de lavar roupas. Sob a ótica da linguagem gráfica, foi delineado um estudo analítico que compreende o desenvolvimento de um protocolo de análise gráfica de interfaces, bem como a seleção e análise de exemplares de interfaces de máquinas de lavar roupas. Por meio desse estudo analítico, busca-se apresentar sugestões para o desenvolvimento de um modelo conceitual de interface com abordagem do Design Inclusivo.

\section{Linguagem gráfica em interfaces}

A interface como mediadora entre sistema e usuário opera diretamente por meio de componentes gráficos básicos (Campos et al, 2008) que, integrados, compõem unidades comunicacionais da linguagem visual. Para Twyman (1982) a linguagem gráfica é apresentada a partir de três modos de simbolização: verbal, pictórica (marcas compreendidas como ilustrações) e esquemática (marcas não verbais ou pictóricas).

A relação entre elementos gráficos a partir dessas formas de simbolização desperta associações semânticas inerentes à cognição humana, representadas por variáveis gráficas (Mijksenaar, 1997).

Para o modelo de Mijksenaar (1997), essas variáveis são agrupadas segundo as associações que despertam. Variáveis hierárquicas provêm diferenciação de graus de importância, via tamanho e intensidade; e variáveis diferenciadoras indicam diferenças tipológicas, via cores e formas (Quintão \& Triska, 2014). Elas podem ainda ser classificadas como variáveis de suporte, quando possuem função de apoio e de organização (e.g. áreas de cor, linhas, boxes) (Quadro 1). 
Quadro 1: Variáveis gráficas. Fonte: as autoras (2021) com base em Mijksenaar (1997).

\begin{tabular}{l}
\hline Diferenciadoras \\
\hline Cor \\
Ilustrações \\
Largura da coluna \\
Tipografia \\
\hline Hierárquicas \\
\hline Posição sequencial \\
Posição na página (layout) \\
Tamanho da fonte \\
Peso da fonte \\
Espaçamento entrelinhas \\
\hline Súmorte
\end{tabular}

De forma complementar, para Campos et al (2008), as variáveis despertam, por meio da articulação semântica entre elementos gráficos base, o caráter mediador provido por interfaces gráficas. Para tanto, propõem as variáveis por meio de critérios de análise de interfaces gráficas. Dentre elas, destacam-se para este estudo as seguintes:

- Cor: definida por Mijksenaar (1997) como uma variável diferenciadora, a variação cromática permite criar distinção entre superfícies (Campos et al, 2008), o que em interfaces assume papel chave na organização visual. Assim, a análise atenta para quantificar cores. Ao se considerar o uso de cor em áreas de cor e sombreamento, a variável assume papel de suporte (Mijksenaar, 1997);

- Tipografia: considerando os atributos intrínsecos e extrínsecos ao tipo (Twyman, 1982), a tipografia pode assumir caráter diferenciador (e.g. variações e estilos), hierárquico (peso e tamanho) e de suporte (por meio de atributos do texto, itálico e versalete) (Mijksenaar, 1997), sendo fundamental para a organização da informação verbal na interface;

- Contraste cromático: remete à legibilidade e espaçamento de caracteres e figuras sob a perspectiva de cor e, para tipografia, o corpo de fonte utilizado. O contexto da 
pesquisa propicia um diálogo do contraste com os princípios de acessibilidade impressa (escala de Michelson) e digital (taxa de contraste web).

- Para mídia impressa: segundo a Deutscher Blinden und Sehbehindertenverband (DBSV, n.d. $)^{1}$, um contraste acessível não deve ser inferior à 0,7 na escala de Michelson;

- Para mídia digital: segundo a WCAG 2.1 (W3C, 2018), um contraste de cor suficientemente acessível para texto de até 14 pontos é igual ou superior à taxa de 4,5:1 (Michelson= 0,6). Para elementos pictóricos e esquemáticos, a taxa deve ser superior a 3:1;

- Composição: considera simetria e forma na composição como um todo. Essa variável pode ser ainda complementada por posição no layout (Mijksenaar, 1997), a fim de reafirmar o caráter hierárquico da categoria;

- Consistência: para Campos et al (2008), a consistência implica em padrões visuais repetitivos em termos de cores, tipografia, ilustrações (variáveis diferenciadoras (Mijksenaar, 1997) e forma (e.g. botões e visores; variável de suporte (Mijksenaar, 1997);

- Densidade informacional: diz respeito à quantidade de blocos de unidade visual presentes na interface.

As categorias descritas anteriormente podem assumir caráter descritivo (e.g. quantidade) ou objetivo (e.g. consta ou não consta) conforme cada critério que contempla (Figura 2).

\footnotetext{
${ }^{1}$ Associação Alemã de Cegos e Deficientes Visuais

${ }^{2}$ Web Accessibility in Mind: organização sem fins lucrativos do Center for Persons with Disabilities (Utah State 
Figura 2: Relação de categorias agrupadas por tipo de resposta obtida. Fonte: as autoras (2021).

\section{Categorias descritivas}

\section{Cor}

Quantidade de cores impressas

Quantidade de cores luminosas

\section{Tipografia \\ Corpo (aproximado) no impresso}

Densidade informacional

Quantidade de blocos de informação

\section{Categorias objetivas}

Cor
Área de cor e sombreamento
Tipografia
Serifa
Bold
Itálico
Caixa alta

\section{Contraste cromático}

Elementos gráficos (impresso)

Tipografia (impresso)

Elementos gráficos (digital)

Tipografia (digital)

\section{Composição \\ Posição no layout \\ Simetria \\ Forma \\ Consistência \\ Identificação por cor \\ Identificação por forma \\ Identificação por pictograma \\ Identificação por texto}

De forma paralela à análise de variáveis, a fim de abranger amplamente os estudos sobre a linguagem gráfica utilizada nas interfaces, Twyman (1979, p.121) sugere a investigação sobre "métodos de configuração" gráfica. Essas configurações determinam a organização estrutural de como a informação é apresentada aos usuários, o que influencia no modo como a compreendem, interagem e "navegam" por ela. Dessa maneira, mapeá-las oferece embasamentos sobre as estratégias perceptivas de como as interfaces atuam na mediação.

Em termos analíticos, Twyman (1979) define uma matriz de estudo das configurações em torno da linearidade da informação. Para o autor, esse direcionamento é crítico para compreender as estratégias de conciliação entre linguagens comumente lineares (como a verbal) e não lineares (como a pictórica). Ademais, diferentes configurações implicam também 
em rompimentos diferentes com a linearidade, por meio de quebras semânticas e mecânicas, o que pode inferir graus de significado à estratégia das interfaces. Como explica Horn (1998), essas configurações correspondem à diversidade com a qual a visão pode ser direcionada sobre a informação.

Conforme a Figura 3, nas linhas da matriz encontram-se os modos de simbolização (verbal numérico, pictórico e verbal numérico, pictórico, esquemático), enquanto que nas colunas encontram-se os modos de configuração (Twyman, 1979). São eles:

- Linear puro: informação linear sem quebras (e.g. a informação textual contínua apresentada em torno de um botão rotativo de seleção);

- Linear interrompido: normalmente uma única unidade semântica, com quebras intencionais ou não (e.g. a organização da informação textual de um visor digital);

- Lista: cada linha é uma unidade semântica própria (e.g.um menu suspenso);

- Linear ramificado: representação com ramificações (e.g. um menu suspenso com submenus);

- Matriz: layout criado por dados organizados em colunas e linhas (e.g. Figura 3);

- Não-linear dirigido: direciona o usuário para uma informação (e.g. ordem de leitura ocidental, direita para esquerda, de cima para baixo);

- Não-linear aberto: não direcional (e.g. informações organizadas de forma livre).

Figura 3: Matriz de Twyman (1979). Fonte: as autoras (2021).

\begin{tabular}{|c|c|c|c|c|c|c|c|}
\hline & $\begin{array}{l}\text { Linear } \\
\text { puro }\end{array}$ & $\begin{array}{l}\text { Linear } \\
\text { interrompido }\end{array}$ & Lista & $\begin{array}{l}\text { Linear } \\
\text { ramificado }\end{array}$ & Matriz & $\begin{array}{l}\text { Não-linear } \\
\text { dirigido }\end{array}$ & $\begin{array}{l}\text { Não-linear } \\
\text { aberto }\end{array}$ \\
\hline $\begin{array}{l}\text { Verbal/ } \\
\text { numérico }\end{array}$ & & & & & & & \\
\hline $\begin{array}{l}\text { Pictórico } \\
\& \text { Verbal/ } \\
\text { numérico }\end{array}$ & & & & & & & \\
\hline Pictórico & & & & & & & \\
\hline Esquemático & & & & & & & \\
\hline
\end{tabular}

Como alerta Twyman (1982), as categorias propostas não são necessariamente discretas. Ou seja, classificações podem ser subjetivas, e estão sujeitas não apenas à compreensão de quem analisa, mas à ambiguidade contida em muitas formas de apresentação visual (Twyman, 1982, p.119).

Os elementos físicos e variáveis gráficas, bem como os modos de simbolização e configuração presentes na interface, são primordiais para a construção da análise gráfica nas 
etapas seguintes da pesquisa. A articulação entre diferentes modelos faz-se necessária a fim de abranger múltiplas dimensões das amostras, e mapear pontos de atenção condizentes com os objetivos da pesquisa como um todo. Para isso, é imperativo também compreender a configuração de comandos e funcionalidades nas interfaces de eletrodomésticos e sua transposição para a acessibilidade.

Diante de pesquisas na área de acessibilidade de eletrodomésticos (Aguiar, 2004; Rezende, 2014; Raposo, 2015; Oliveira, 2018), identifica-se que a interface da máquina de lavar roupas compreende um conjunto de comandos de ação e seleção visuais que possibilitam a realização da tarefa de lavar roupas. A acessibilidade desses comandos é questionada devido à complexidade da apresentação da interface. De modo geral, este eletrodoméstico apresenta um sistema eficiente que controla a velocidade e a performance de lavagem conforme $o$ programa selecionado (e.g. a seleção pode ocorrer de acordo com o tipo de tecido, nível de sujeira, cor ou temperatura de lavagem, indicados nas etiquetas das roupas) (Adam, 2021; Aguiar, 2004).

A apresentação visual dos comandos pode variar de acordo com a apresentação da informação (e.g. verbal, pictórica e esquemática), as categorias descritivas e objetivas dos componentes gráficos (e.g. cor, densidade informacional, tipografia, contraste, consistência, composição), e suas características funcionais (e.g. ação, seleção) (Campos et al, 2008; lida, 2005; Mijksenaar, 1997; Twyman, 1982). Já a apresentação física dos comandos (controles) pode ser classificada, de acordo com lida (2005), conforme sua função de posicionamento, podendo ser:

- controle discreto, quando assume posições bem definidas: botão físico ou digital, teclas, botão rotativo com número limitado de posições;

- controle contínuo, quando permite diversos ajustes: botão rotativo com posicionamento quantitativo/ intensidade, ex: botão rotativo de volume.

O estudo conduzido por meio do protocolo propicia investigar de forma integrada diferentes aspectos da apresentação da linguagem gráfica nas interfaces, e relacioná-los às funções disponíveis nos eletrodomésticos.

\section{Metodologia}

Este estudo teórico-analítico compreende o desenvolvimento de um modelo de análise de componentes de interfaces de eletrodomésticos, seguido de uma análise gráfica de interfaces de máquinas de lavar roupas. $O$ estudo tem o objetivo de identificar os componentes gráficos e suas relações funcionais na estrutura da interface com a finalidade de identificar um padrão gráfico-informacional para interfaces de máquinas de lavar roupas (Figura 4). 
Figura 4: Procedimentos metodológicos. Fonte: as autoras (2021).

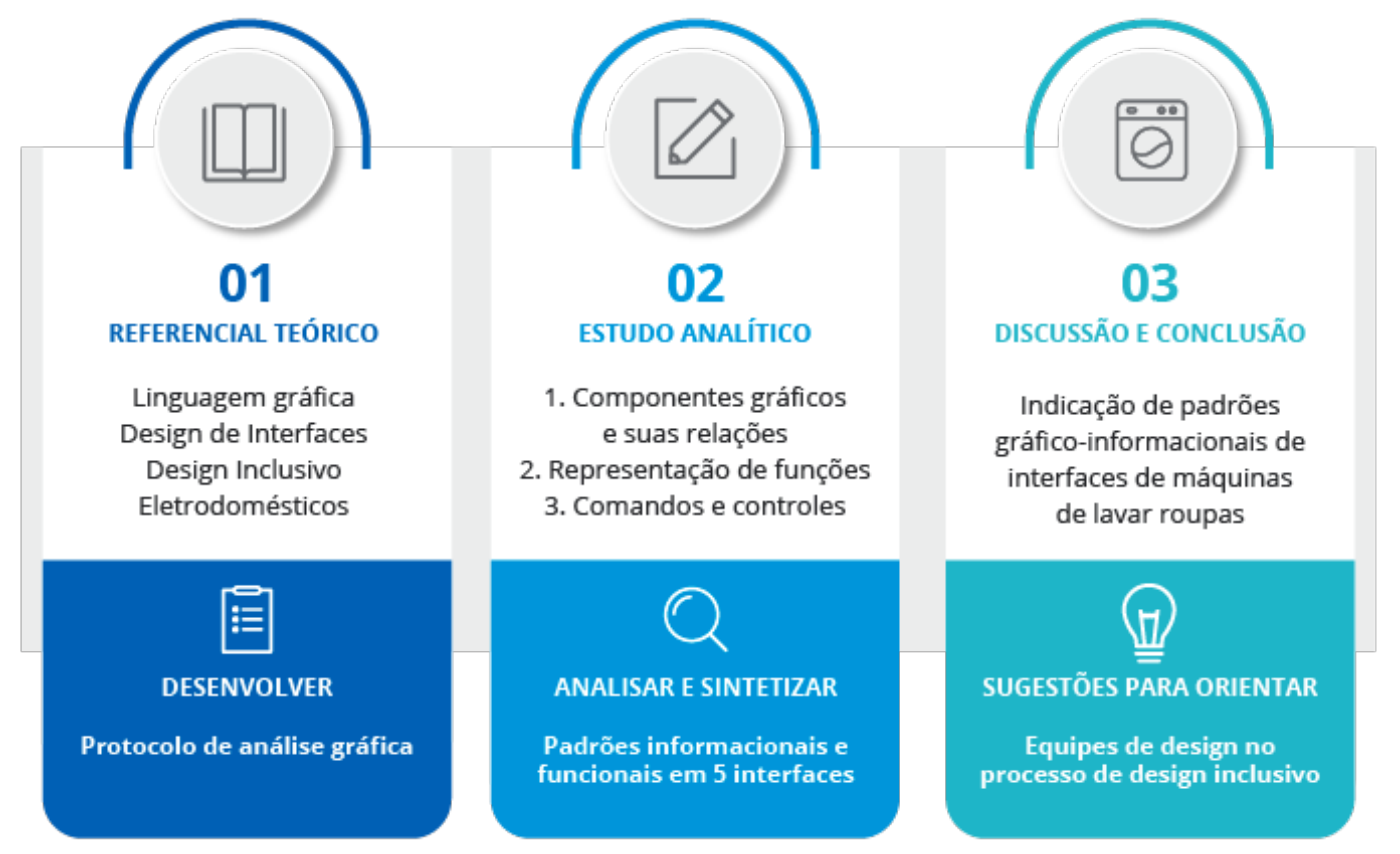

A partir do referencial teórico sobre linguagem gráfica e interfaces, foi organizado um protocolo de análise, com o intuito de estabelecer uma relação semântica entre os componentes informacionais e funcionais da interface. O protocolo de análise foi organizado em três seções, sob a perspectiva da linguagem gráfica (Campos et al, 2008; Mijksenaar, 1997; Twyman, 1982).

A seção 1, "Componentes gráficos e relações", foi direcionada para o levantamento de componentes gráficos e das relações estabelecidas entre eles, a partir dos critérios descritivos sobre interfaces propostos por Campos et al (2008), complementado por Mijksenaar (1997) e Twyman (1982). Para essa etapa, buscou-se investigar as interfaces como um todo integrado em termos gerais de apresentação, a partir de categorias descritivas (e.g. quantidade de cores luminosas e impressas) e objetivas (e.g. consta ou não consta).

A seção 2, "Representação de funções", buscou investigar a configuração dos elementos verbais, pictóricos e esquemáticos, especificamente, a partir da matriz descritiva de Twyman (1979). Nessa etapa, foram analisadas as formas de representação de funcionalidades específicas de máquinas de lavar, já então mapeadas.

A seção 3, "Comandos e controles", de cunho técnico, foi direcionada para investigar a presença e apresentação dos componentes físicos da interface, a partir de três categorias: visor LED, comandos de ação e comandos de seleção (lida, 2005). A análise buscou apontar a presença desses elementos, e descrever brevemente suas características (e.g. cor de feedback luminoso). As três seções visam relacionar os componentes gráficos e funcionais da interface, com o intuito de identificar uma tendência para ser posteriormente comparada aos requisitos de acessibilidade apresentados pelo WCAG 2.1 (W3C, 2018). A Figura 5 ilustra o protocolo desenvolvido. 
Figura 5: Protocolo de análise gráfica de interfaces de máquina de lavar roupas. Fonte: as autoras (2021).

SEÇÃO 1 - Componentes gráficos e relações

\begin{tabular}{|c|}
\hline Cor \\
\hline Quantidade de cores impressas \\
\hline Quantidade de cores luminosas \\
\hline Área de cor e sombreamento \\
\hline Tipografia \\
\hline Corpo (aproximado) no impresso \\
\hline Serifa \\
\hline Bold \\
\hline Itálico \\
\hline Caixa alta \\
\hline Contraste cromático \\
\hline Elementos gráficos (impresso) \\
\hline Tipografia (impresso) \\
\hline Elementos gráficos(digital) \\
\hline Tipografia (digital \\
\hline Composição \\
\hline Posição no layout \\
\hline Simetria \\
\hline Forma \\
\hline Consistência \\
\hline Identificação por cor \\
\hline Identificação por forma \\
\hline Identificação por pictograma \\
\hline Identificação por texto \\
\hline Densidade informacional \\
\hline
\end{tabular}

Quantidade de blocos de informação
SEÇÃO 2 - Representação de funções

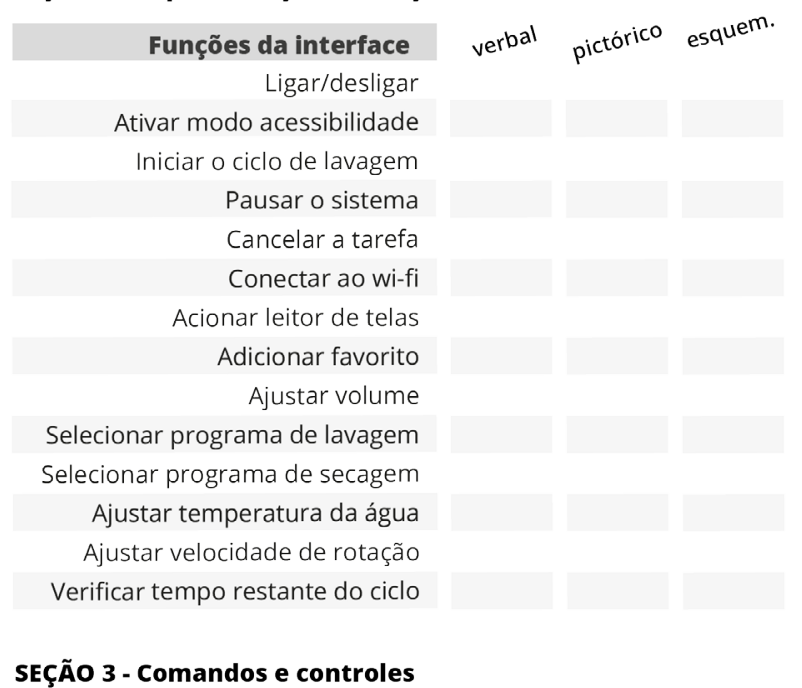

SEÇÃO 3 - Comandos e controles

\begin{tabular}{|r|r|}
\hline Visor LED & Comandos de seleção \\
\hline Sinal de luz & Botão com relevo \\
\hline Botão touchscreen & Botão sem relevo \\
\hline Comandos de ação & Botão rotativo \\
\hline Botão com relevo & Feedback luminoso \\
Botão sem relevo & \\
Botão rotativo & \\
\hline Feedback luminoso & \\
\hline
\end{tabular}

Para a análise, foram selecionadas cinco interfaces de máquinas de lavar roupas disponíveis no mercado brasileiro. Duas possuem abertura superior e três abertura frontal, uma distinção necessária visto que essa disposição pode ter impacto sobre o modo de funcionamento da interface frente às funções táteis (e.g. levantar a tampa). Considera-se que, este número de interfaces analisadas é condizente, pois em análise gráfica prévia, as demais interfaces encontradas no mercado brasileiro seguem os padrões gráfico-informacionais das interfaces aqui apresentadas nesta análise.

Devido ao contexto pandêmico, foi necessário analisar as interfaces via registros fotográficos disponibilizados oficialmente pelas fabricantes. As figuras 6 e 7 representam as interfaces analisadas. 
Figura 6: Representações das interfaces de máquinas com abertura superior. Fonte: as autoras (2021).
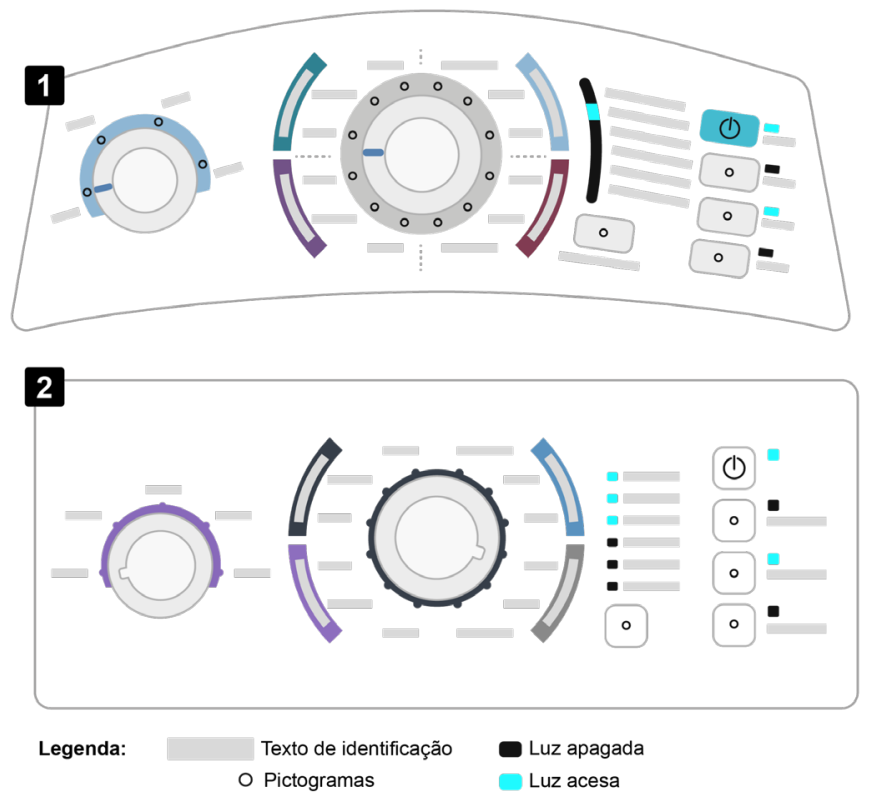

Figura 7: Representações das interfaces de máquinas com abertura frontal. Fonte: as autoras (2021).
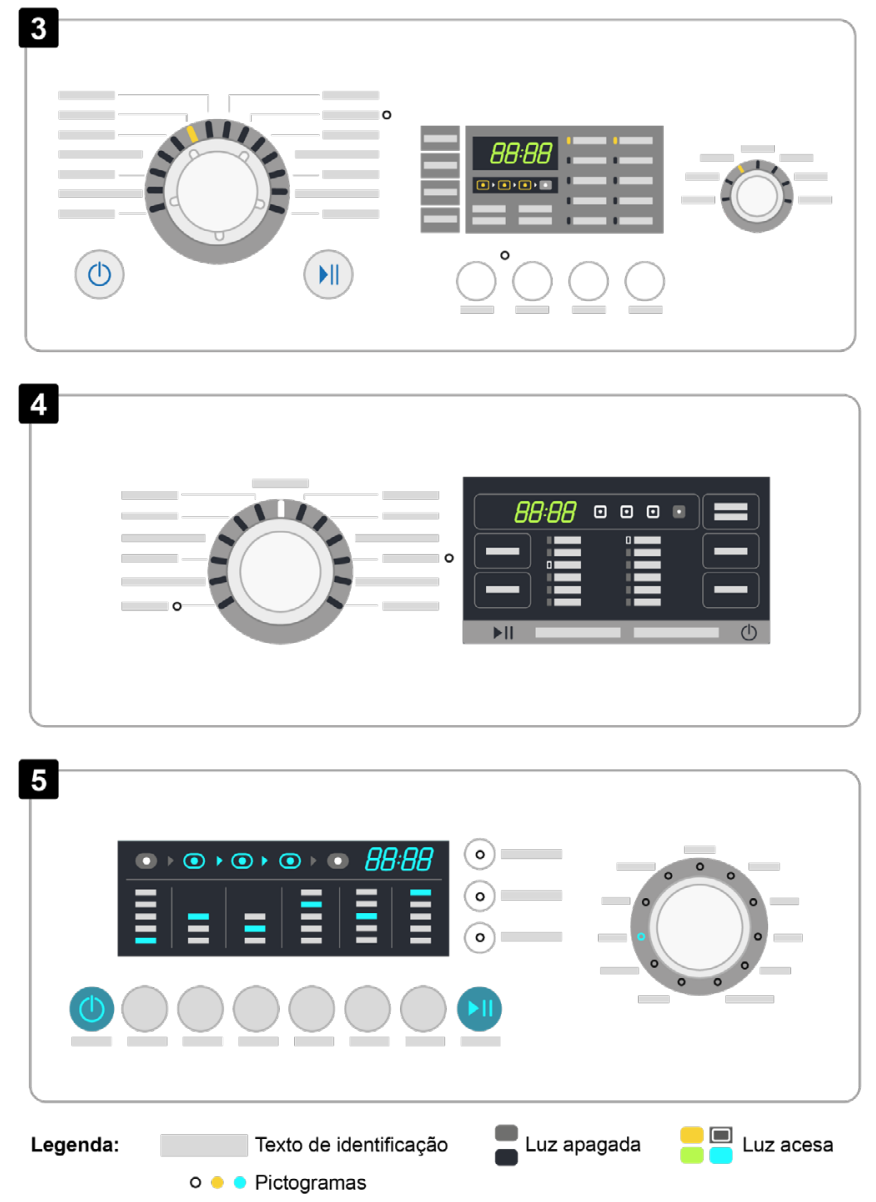
A análise das interfaces foi realizada a partir da identificação visual dos componentes gráficos, funções e representações com base no protocolo desenvolvido.

Na seção 1, dentro das categorias descritivas, cada variável foi analisada da seguinte forma:

- Cor: as cores da interface como um todo foram quantificadas. Para cores impressas não foram contabilizadas as cores de escala de cinza (cinzas, branco e preto). Optouse por não realizar diferenciação entre tons de cinza em geral, devido à proximidade que alguns desses aparentavam mediante a foto das interfaces. Dessa forma, a fim de analisar criteriosamente cada uma dessas tonalidades, seria necessário ter contato presencial com cada interface;

- Tipografia: foram considerados apenas os elementos tipográficos utilizados para identificar funções e modos de operação, que compunham a maior parcela de informação verbal. O corpo da tipografia foi estimado a partir das dimensões da interface, dos textos individuais e da fonte utilizada, e configura um número (em pontos) aproximado;

- Densidade informacional: os blocos de informação foram identificados a partir de unidade visual (similaridade), posição e espaçamento no layout, de agrupamento das informações e das diferenças entre ordem de leitura em cada seção.

Dentro das categorias objetivas (Seção 1), apenas a categoria "contraste cromático" contou com uma análise também qualitativa (atende ou não atende), tendo como base as taxas de contraste mínimas trazidas no referencial teórico. Devido às restrições da pesquisa causadas pela pandemia, somente foi possível medir o contraste a partir de fotografias em suporte digital, por meio da ferramenta de verificação de contraste digital da WebAIM ${ }^{2}$. Ainda que as checagens tenham sido feitas em duplicata, ressalta-se que as condições de análise das cores impressas e digitais podem proporcionar certos desvios nas medições. Ademais, as demais categorias da seção 1 foram analisadas com base nos critérios definidos por Campos et al (2008) e Mijksenaar (1997).

Os resultados da análise foram então tabulados, o que permitiu identificar a recorrência dos padrões conforme delimitado em cada seção. Os elementos indicados na síntese são aqueles que constavam em três ou mais amostras, representando assim a maioria delas.

\section{Resultados e discussão}

A seguir são apresentados os resultados das análises das interfaces de máquina de lavar roupas, segmentados em seções, conforme o protocolo desenvolvido.

A análise da seção 1 "Componentes gráficos e relações" revelou que, frente ao uso de cor impressa, todos os artefatos empregam cores além da informação em preto e/ou branco, em uma

\footnotetext{
${ }^{2}$ Web Accessibility in Mind: organização sem fins lucrativos do Center for Persons with Disabilities (Utah State University).
} 
média de uma a três cores adicionais. Esse dado soma-se à tendência de todos apresentarem área de cor e sombreamento, revelando assim que essa variedade cromática pode ter implicações sobre a diferenciação visual e organização hierárquica da interface (Mijksenaar, 1997).

Por outro lado, apesar de todas as amostras apresentarem alguma fonte luminosa com cor, verifica-se uma menor variação cromática: uma a duas cores. Nas máquinas de abertura frontal, uma cor costuma ser estabelecida como principal, enquanto outras sinalizam dados secundários que requerem diferenciação. No entanto, não foi observada uma padronização nos elementos que eram contemplados pela cor principal ou pelas secundárias.

Nota-se ainda a utilização de cor junto a elementos verbais, pictóricos e esquemáticos (e.g. área de sombreamento), o que é condizente com diretrizes de acessibilidade básica diferenciação, visto que cor não deve ser utilizada como o único recurso visual para diferenciar ou transmitir informação (W3C, 2018).

Já para a tipografia impressa, observa-se que os blocos com quantidade mais expressiva de informação escrita possuem, aproximadamente, um corpo mínimo de 11 pontos. No geral, há uma priorização pelo uso de uma única família tipográfica não serifada, o que contribui para a consistência da interface. Os principais atributos do texto mapeados incluem o uso de alternância no peso da fonte, bem como informações somente em caixa alta, o que configura atributos hierárquicos e de suporte da informação textual (Mijksenaar, 1997). Entretanto, devido à alternância no uso do peso e do tamanho de diferentes níveis hierárquicos de texto nas interfaces, são necessários estudos futuros quanto à acuidade visual dos elementos textuais. Julga-se que esse aprofundamento é necessário para relacionar os resultados diretamente às recomendações de acessibilidade.

De modo geral, as interfaces tendem a apresentar taxas de contraste suficientes para identificação dos componentes gráficos, tanto para texto quanto para elementos não-textuais. Dentre as três amostras que apresentavam visor LED, apenas uma não apresentou contraste digital (em texto e outros elementos) suficiente. No entanto, questiona-se se o contraste é suficiente para permitir a acessibilidade para pessoas com deficiência visual, por exemplo, visto que, apesar de as taxas obtidas serem estimadas, todas as interfaces apresentaram ressalvas quanto a contraste e legibilidade (DBSV, n.d.; WebAIM, n.d.; W3C, 2018).

Considerando a composição gráfica dos elementos na interface, as amostras como um todo apresentam posição no layout e simetria como artifícios organizacionais. Essas estratégias de organização tornam-se primordiais para hierarquizar as informações (Mijksenaar, 1997), considerando que os artefatos tendem a apresentar, em média, de dois a três blocos de informação.

Enfim, considerando os artifícios utilizados para manter a consistência da interface, identifica-se que os artefatos mantêm uma unidade visual na apresentação dos componentes gráficos de identificação, visto que apresentam repetição dentro dos blocos (Campos et al, 2008). A unidade textual e por forma foram as mais predominantes, respectivamente na identificação de modos (e.g. modos de lavagem e secagem) e no formato de botões e teclas.

A Figura 8 apresenta uma síntese dos resultados da seção 1. 
Figura 8: Síntese de resultados da seção 1 "Componentes gráficos e relações”. Fonte: as autoras (2021).

\begin{tabular}{|c|c|}
\hline \multicolumn{2}{|l|}{ Cor } \\
\hline Quantidade de cores impressas & 1 a 3 \\
\hline Quantidade de cores luminosas & 2 cores \\
\hline Área de cor e sombreamento & • \\
\hline \multicolumn{2}{|l|}{ Tipografia } \\
\hline Corpo (aproximado) no impresso & $11 \mathrm{pts}$ \\
\hline \multicolumn{2}{|l|}{ Serifa } \\
\hline Bold & • \\
\hline \multicolumn{2}{|l|}{ Itálico } \\
\hline Caixa alta & - \\
\hline \multicolumn{2}{|l|}{ Contraste cromático } \\
\hline Elementos gráficos (impresso) & - \\
\hline Tipografia (impresso) & - \\
\hline Elementos gráficos(digital) & - \\
\hline Tipografia (digital & - \\
\hline \multicolumn{2}{|l|}{ Composição } \\
\hline Posição no layout & - \\
\hline Simetria & - \\
\hline \multicolumn{2}{|l|}{ Forma } \\
\hline \multicolumn{2}{|l|}{ Consistência } \\
\hline Identificação por cor & - \\
\hline Identificação por forma & - \\
\hline Identificação por pictograma & - \\
\hline Identificação por texto & - \\
\hline \multicolumn{2}{|l|}{ Densidade informacional } \\
\hline Quantidade de blocos de informação & 2 a 3 \\
\hline
\end{tabular}

Já a seção 2 "Representação de funções" revelou que as interfaces das máquinas de lavar roupas apresentam o uso expressivo de linguagem verbal e esquemática. Enquanto isso, a linguagem pictórica é reservada principalmente para identificação de funções de seleção (e.g. programa de lavagem e secagem), porém sem aparentes convenções quanto a situações de uso. Ressalta-se que, quando presente, acompanha elementos verbais, podendo se enquadrar na classificação de linguagem pictórica-verbal de Twyman (1979). Ademais, esse uso complementar é condizente com diretrizes de acessibilidade básica, que estipulam que funções e comandos que exigem input identificados por elementos não-textuais requerem também identificação verbal descritiva.

Em contrapartida, a linguagem verbal se mostra diversa. Em comandos de ação assume principalmente as configurações linear puro e linear interrompido. A quebra na linearidade propiciada pela diferença entre um modo e outro ocorre tanto por ergonomia, para separar funções distintas atreladas ao mesmo comando (e.g. botão de ligar e desligar), o que configura também uma separação semântica; quanto mecanicamente, para adequação aos limites físicos da interface e do layout do bloco (Twyman 1979) (Figura 9). 
Figura 9: Quebra semântica para separação de funções (à esquerda), e quebra mecânica para adequação ao layout (à direita). Fonte: as autoras (2021).
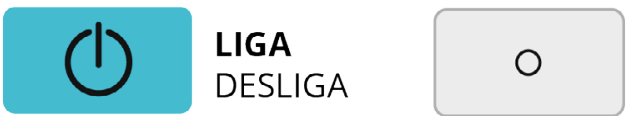

DUPLO

ENXÁGUE

Em comandos de seleção (e.g. programa de lavagem e secagem), predomina a

configuração de lista, visto que cada rótulo textual é uma unidade semântica própria (Twyman, 1979). Entretanto, devido à disposição desses elementos (em torno de botões rotativos circulares, organizados de forma convergente ao centro), é também possível considerar uma configuração não-linear aberta, caso considerada a informação puramente textual. Novamente, a identificação verbal favorece a acessibilidade da interface (W3C, 2018). Ressalta-se, ainda, que a disposição em lista propicia que rótulos verbais atendam imediatamente às diretrizes de acessibilidade referentes à largura da coluna de texto (e.g. não maior que 80 caracteres).

É importante ressaltar que essa informação verbal vem frequentemente acompanhada de elementos esquemáticos de suporte, como áreas de sombreamento e linhas. Nas interfaces, esses elementos são principalmente configurados em não-linear dirigido, o que vem de encontro com sua função de apoio por direcionar o usuário para a informação verbal (Mijksenaar, 1997). Em funções de seleção, a informação esquemática pode ainda assumir a configuração de lista, se considerada isoladamente (Figura 10). A utilização desses suportes esquemáticos propicia que rótulos verbais sejam alinhados às margens pela esquerda ou pela direita, a depender da posição em relação ao botão circular, o que condiz com orientações de acessibilidade (W3C, 2018).

Figura 10: Recorte de botão rotativo de seleção de modos de lavagem. Os elementos esquemáticos (linhas e marcadores) se configuram como não-linear dirigido ao darem suporte para a informação verbal. Fonte: as autoras (2021).

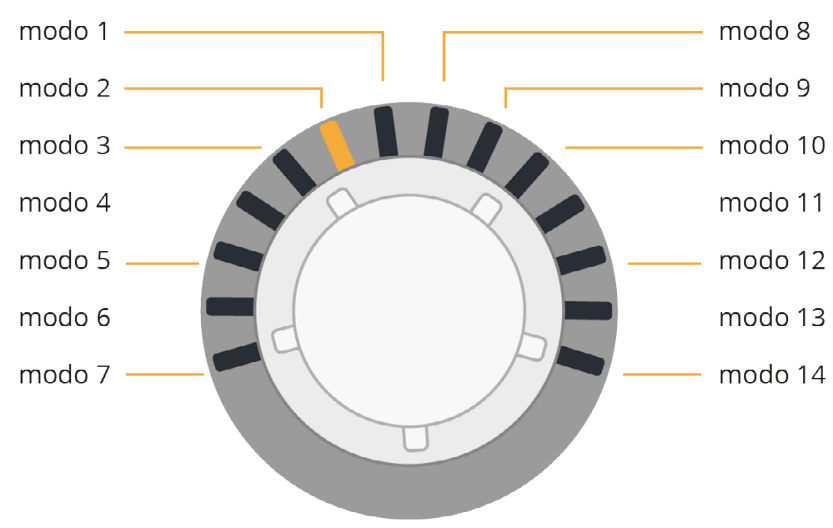

Para ambos os casos, é importante ressaltar novamente a subjetividade das categorias de Twyman (1979), o que pode requerer discussões mais aprofundadas sobre os resultados obtidos. A segunda seção ainda permitiu notar a ausência em todas as amostras de funções relacionadas à acessibilidade da interface, como leitor de telas e ajuste de volume.

A Figura 11 apresenta uma síntese dos resultados da seção 2. 
Figura 11: Síntese de resultados da seção 2 "Representação de funções". Fonte: as autoras (2021).

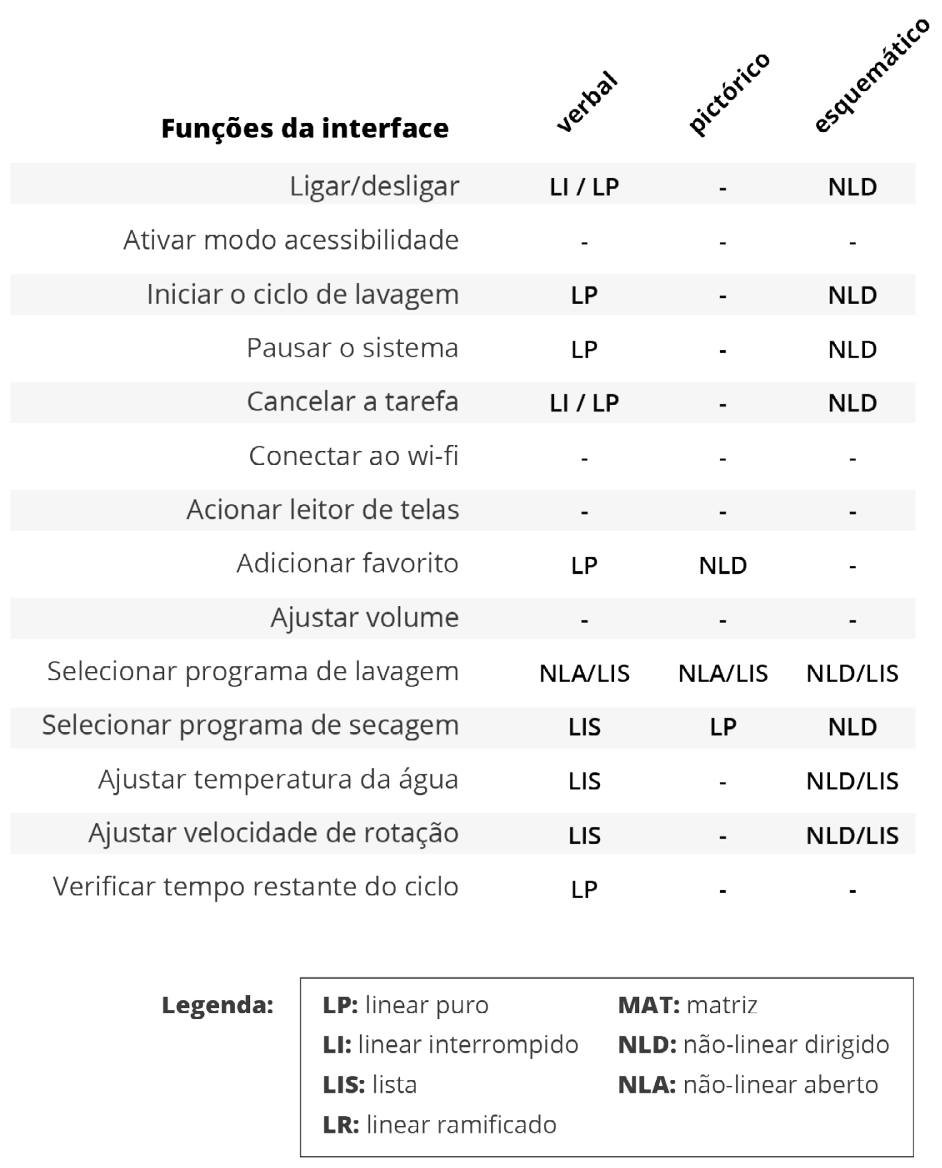

Por fim, os resultados da seção 3 "Comandos e controles" mostram que as três categorias de funções - visor LED, comandos de ação e de seleção - estão presentes na maior parte das amostras. Visores LED, entretanto, somente foram apresentados por aquelas máquinas com abertura superior. Nessas, por sua vez, o visor é utilizado principalmente para dispor informações luminosas, e não tende a contar com interação touchscreen.

Aponta-se ainda que comandos de ação tendem a ser apresentados a partir de botões com relevo, com formato retangular $(n=2)$ ou circular $(n=2)$ e atrelados a algum tipo de feedback luminoso na interface, de cor azul $(n=2)$ ou âmbar $(n=2)$, sendo classificados como comandos discretos, conforme lida (2005).

Já comandos de seleção, presentes em todas as amostras, tendem a utilizar botões rotativos circulares, também atrelados a feedback luminoso na cor azul $(n=3)$, branca $(n=1)$ ou âmbar $(n=1)$. Estes controles são classificados como rotativos discretos com posições definidas (indicativo luminoso - led), conforme lida (2005). Enquanto não parece existir uma padronização frente ao uso de cor luminosa, indica-se que são necessárias investigações futuras sobre a arbitrariedade dessas características. A Figura 12 apresenta a síntese dos resultados da seção 3 . 
Figura 12: Síntese de resultados da seção 3 "Comandos e controles". Fonte: as autoras (2021).

\begin{tabular}{|r|r|r|}
\hline Visor LED & - & \\
Sinal de luz & $\bullet$ & \\
\hline Botão touchscreen & & \\
\hline Comandos de ação & - & \\
\hline Botão com relevo & $\bullet$ & retangular | circular \\
Botão sem relevo & & \\
Botão rotativo & & \\
\hline Feedback luminoso & - & azul | âmbar \\
\hline Comandos de seleção & - & \\
\hline Botão com relevo & & \\
Botão sem relevo & & \\
Botão rotativo & $\bullet$ & circular \\
\hline Feedback luminoso & - & azul | âmbar | branca \\
\hline
\end{tabular}

A partir do desenvolvimento do protocolo de análise e estudo analítico é possível inferir que as interfaces gráficas de máquinas de lavar roupas apresentam:

- Utilização de cores como componente de diferenciação hierárquica da informação, bem como em atributos de suporte por meio de elementos esquemáticos;

- Apresentação tipográfica com mínimo de 11 pontos, em média com apenas uma família tipográfica. Atributos de texto de peso e caixa alta são utilizados como artifícios hierárquicos e de suporte;

- Contraste visualmente perceptível entre componentes da interface que, no entanto, pode não ser suficiente para atender aos usuários com baixa visão;

- Estrutura informacional equilibrada e simétrica, trazendo uma densidade informacional de 2 a 3 blocos;

- Boa consistência, ocasionada por repetição em componentes gráficos de identificação, especialmente aqueles textuais e de forma;

- Linguagem pictórica utilizada para identificação de funções, porém sem padronização na situação de uso;

- Elementos pictóricos e esquemáticos com função de suporte;

- Comandos de ação configurados pela linguagem verbal de forma linear e comandos de seleção em lista, com elementos esquemáticos não-lineares dirigidos;

- Comandos de ação em relevo (e.g. ligar, pausar), com feedback luminoso;

- Comandos de seleção rotativos circulares com feedback luminoso (e.g. seleção do programa lavagem).

Frente aos resultados descritivos referentes à análise gráfica das interfaces, bem como sua integração aos comandos disponíveis, é possível averiguar que determinadas configurações e utilizações complementares de modos da linguagem gráfica favorecem diretamente a acessibilidade. Os principais pontos encontrados nesse quesito encontram-se compilados no Quadro 2 a seguir: 
Quadro 2: Relação entre apresentação da linguagem gráfica e recomendações de acessibilidade. Fonte: as autoras (2021), com base nas diretrizes WCAG (W3C, 2018).

\begin{tabular}{ll}
\hline Apresentação da linguagem gráfica & Recomendação de acessibilidade atendida \\
\hline $\begin{array}{l}\text { Utilização de cor como recurso de diferenciação, } \\
\text { porém também como de suporte a outros } \\
\text { elementos visuais; }\end{array}$ & $\begin{array}{l}\text { Cor não é o único recurso visual utilizado para } \\
\text { transmitir informação; }\end{array}$ \\
$\begin{array}{l}\text { Funções identificadas de forma complementar } \\
\text { por linguagem pictórica e verbal; }\end{array}$ & $\begin{array}{l}\text { Input identificado por elementos não-textuais } \\
\text { requerem identificação verbal; }\end{array}$ \\
$\begin{array}{l}\text { Programas de seleção identificados por rótulos } \\
\text { verbais curtos dispostos em lista; }\end{array}$ & Colunas de texto com largura acessível; \\
$\begin{array}{l}\text { Rótulos verbais no comando de seleção } \\
\text { acompanhados por elementos esquemáticos de } \\
\text { suporte (e.g. linhas conectoras). }\end{array}$ & $\begin{array}{l}\text { Rótulos verbais (não justificados) alinhados à } \\
\text { esque à direita. }\end{array}$ \\
\hline
\end{tabular}

\section{Considerações finais}

O presente estudo teve o objetivo de analisar sob a ótica da linguagem gráfica, os componentes visuais de interfaces de máquinas de lavar roupas. Por meio do protocolo de análise foi possível identificar uma tendência de uso de componentes visuais verbais, pictóricos e esquemáticos, bem como suas relações informacionais e funcionais na interface.

De modo geral, a investigação evidencia que a utilização da linguagem gráfica em interfaces de máquinas de lavar roupas é flexível. Porém, é imperativo considerar as expectativas de quem projeta frente os modos de utilização do usuário, e como ele vai interagir com a linguagem gráfica. Essa questão se torna especialmente importante considerando o contexto de acessibilidade, considerando a construção de interfaces com linguagem acessível a pessoas com e sem deficiência visual, e em diversos níveis.

O protocolo desenvolvido buscou abranger as relações e configurações da linguagem visual gráfica presente nas interfaces, e se mostrou efetivo para esse fim. Os dados obtidos na análise, tanto objetivos quanto descritivos, propiciaram o apontamento de tendências e, simultaneamente, uma compreensão sobre as implicações que eles trazem sobre a informação.

Entretanto, ressalta-se que as três seções propostas tornam o processo de análise extenso, o que apresentou obstáculos para a revisão dos dados. A subjetividade presente em algumas categorias, e em especial na seção 2 , ainda demandou que todas as análises fossem feitas em um único momento, a fim de manter a coesão dos critérios subjetivos, o que pode apresentar problemas logísticos para eventuais análises futuras e maiores.

Os resultados levantados propiciam compreender as estratégias de design da informação das interfaces, o que implica em formas diferentes de mediação para os usuários. O protocolo desenvolvido busca uma aproximação de estudo relacional entre linguagem gráfica e design de interfaces de eletrodomésticos, a fim de criar uma base de análise sólida para abordar tanto a informação gráfica quanto às funções técnicas dispostas sobre elas. 
A partir deste estudo recomenda-se, para pesquisas futuras, um estudo prático para observar a interação do usuário com e sem deficiência visual com as interfaces analisadas, seguida do desenvolvimento de um modelo conceitual de interface acessível a partir dos resultados apresentados neste artigo. Para tal, é importante considerar que as formas de configuração da linguagem gráfica oferecem múltiplas soluções para abordar a acessibilidade, de forma complementar a outros recursos assistivos nas interfaces.

\section{Agradecimento}

O presente trabalho foi realizado com apoio da Coordenação de Aperfeiçoamento de Pessoal de Nível Superior - Brasil (CAPES) - Código de Financiamento 001.

\section{Referências}

Adam, D. L., \& Okimoto, M. L. L. R. (2021). Acessibilidade de eletrodomésticos sob a ótica da pessoa com deficiência visual: estudo exploratório. In Canal 6 Editora (Ed.), III CBTA Congresso Brasileiro de Tecnologia Assistiva. Canal 6 Editora.

Adam, D. L., Okimoto, M. L. L. R., \& Nascimento, I. Z. (2020). Mapeamento de Recursos Digitais de Tecnologia Assistiva: Interação multimodal para pessoas com deficiência visual e cegas. In A inovação emergente : tecnologias e interfaces. CEGRAF UFG.

Aguiar, V. C. de. (2004). O desenvolvimento da usabilidade de interfaces em projetos - um estudo de caso em lavadora de roupa. Universidade Federal de São Carlos.

Clarkson, J., Coleman, R., Hosking, I., \& Waller, S. (2007). Inclusive design toolkit.

Campos, B. A., Walter, Y., Rodrigues, L. P. S., \& Marar, J. F. (2008). Análise gráfica da interface digital de um sistema de informação para seleção de materiais. UNOPAR Científica Ciências Exatas e Tecnológicas, 7, 37-44.

DBSV (Deutscher Blinden und Sehbehindertenverband). (n.d.). Kontrast und Farben. Leserlich Info. Acesso em 6 de maio de 2021. https://www.leserlich.info/kapitel/farben.php\#empfehlung

Horn, R. E. (1998). Visual Language: Global Communication for the 21st Century (1st ed.). MacroVU Press.

lida, I. (2005). Ergonomia: projeto e produção. Editora Blusher.

Mijksenaar, P. (1997). Visual function: an introduction to information design. Rotterdam: 010 Publishers.

Oliveira, T. A. B. de. (2018). Estímulos sensoriais: Potencialidade na interação de usuários cegos em painéis de eletrodomésticos. Universidade do Estado de Santa Catarina.

Quintão, F. S., \& Triska, R. (2013). Design de informação em interfaces digitais: origens, definições e fundamentos. Revista Brasileira de Design Da Informação, 10(2), 105-118.

Raposo, N. O. (2015). Elementos estruturantes para o projeto de interfaces multimodais. Universidade Federal do Amazonas. 
Rezende, M. H. D. M. (2014). Modelo do projetista e modelo do usuário no design de produtos: um estudo da atividade de lavar roupas. Universidade Federal de São Carlos.

Shneiderman, B., \& Plaisant, C. (2005). Designing the user interface: strategies for effective human-computer interaction (4th ed.). Education, Pearson.

Twyman, M. (1982) The graphic presentation of language. Information Design Journal, 3(1), 2-22.

Twyman, M. (1979). A Schema for the Study of Graphic Language. Kolers, P.A. \& Wrostad, M.E. \& Bouma, H. (Eds.), In: The Processing of Visible Language, 1, Plenum, New York, 117-150.

W3C. (2018). Web Content Accessibility Guidelines (WCAG) 2.1. Web Content Accessibility Guidelines (WCAG) 2.1. https://www.w3.org

WebAIM. (n.d.). WebAIM: Web Accessibility In Mind. WebAIM: Web Accessibility In Mind. Acesso em 26 de abril de 2021. https://webaim.org

\section{Sobre as autoras}

Juliana Ferreira de Oliveira, graduanda, UFPR, Brasil <jubrotx@gmail.com> Dominique Leite Adam, doutoranda PPGDesign e pesquisadora, UFPR, Brasil <domiadam@gmail.com>

Juliana Bueno, Dra., professora e pesquisadora, UFPR, Brasil, <julianabueno.ufpr@gmail.com> Maria Lucia Leite Ribeiro Okimoto, Dra., professora e pesquisadora, UFPR, Brasil <lucia.demec@ufpr.br> 